und für die Küche vielfach verwendbares Produkt liefern. Die relativ großen und sehr harten Steinkerne müssen selbstverständlich entfernt werden. Die unangenehmen Eigenschaften der Marmelade aus Früchten des S, nigra sind bei S. racemosa nicht vorhanden. Wenn auch der Geschmack noch an "Holunder" erinnert, so ist er doch keineswegs aufdringlich, wie bei $\mathrm{S}$. nigra, sondern im Gegenteil angenehm und anregend.

Als Fruchtsaft zubereitet, liefert S. racemosa ein erfrischendes und wohlschmeckendes Getränk. Allerdings darf die „Beigabedosis" nicht zu hoch genommen werden, weil in dieser Verwendungsart der Geschmack zu intensiv ist und daher leicht hervortritt.

Lokal wurde die Marmelade von S. racemosa - wenn auch nur in kleineren Betrieben und in geringem Umfange - als Verfälschungsmittel verwendet.

Die Auswertung zur selbständigen Verarbeitung stößt auf keine Schwierigkeiten, weil das Rohmaterial ja in großen Mengen leicht gesammelt werden kann. Unreife Früchte ergeben ein weniger schmackhaftes Produkt. Im Küchenbedarf gibt die Traubenholundermarmelade jedenfalls eine erwünschte und dankbare Abwechselung.

Die anatomischen Merkmale der Früchte des S. racemosa gleichen denen von S. nigra mit dem Unterschied, dâ der Farbstoff nicht wie bei $\mathrm{S}$. nigra bläulichrot, sondern rötlichgelb ist. An den Fragmenten der Fruchtschale, eventuell an Steinkernfragmenten und an dem Farbstoff ist S. racemosa, auch wo er als Verfälschungsmittel verwendet wird, zia erkennen.

Bezüglich der anatomischen Verbältnisse bei Sambucus nigra verweise ich auf: Tschirch-Österle, Anatomischer Handatlas der Pharmakognosie und Nahrungsmittelkunde (Leipzig 1900, S. 4t) und C. Grie bel, Zur Anatomie einiger einheimischer Früchte, gleichzeitig ein Beitrag zum Vorkommen der sog. Inklusen ${ }^{1}$ ).

Die Marmelade aus den Früchten des Sambucus racemosa soll kein Nachkriegs-Ersatzprodukt sein, sondern, auf Grund ibrer un$\mathrm{zweifelhaften} Q u$ alitäten, eine dankbare und dauernde Bereicherung unserer Nahrungsmittelindustrie.

1) Diese Zeitschrift 1917, $\mathbf{3 3}, 235$.

\title{
Die 1921-er Weinernte in der Pfalz.
}

\author{
Von \\ 0. Krug und G. Fiesselmann. \\ Mitteilung der Landwirtsehaftlichen Kroisversuchsstation Speyer.
}

[Eingegangen am 30. November 1921.]

Das Jahr 1921 wird in der Geschichte des Weinbaues ein denkwürdiges bleiben und durch seine Eigenart eine Sonderstellung einnehmen. Der in der Glut der 1921-er Sonne geborene Neue hat die großen Hoffnungen und Erwartungen der Winzer in bezug auf seine Qualität in vollstem Maße erfüllt, wenn auch die Menge des Frtrags vielfach eine weniger befriedigende war. Sehr hohe Temperatur, sehr viel Sonnenschein, sehr wenig Regen, das waren die charakteristischen Merkmale der Monate, in denen der 192l-er gewachsen und herangereift ist. Dies spiegelt sich auch sehr deutlich wieder in der nachstehenden Tabelle, die ich der Staatlichen Anstalt für Wein- und Obstbau in Neustadt verdanke und die über die Witterungsverhältnisse der Monate Mai bis einschließlich Oktober 1921 Aufschluß gibt. 


\begin{tabular}{|c|c|c|c|c|c|c|c|c|c|}
\hline \multirow{3}{*}{$\begin{array}{c}\text { Monate } \\
1921\end{array}$} & \multicolumn{5}{|c|}{ Sonnenschein } & \multicolumn{2}{|c|}{ Temperatur in ${ }^{\circ} \mathrm{C}$} & \multirow{3}{*}{$\begin{array}{c}\text { Nieder- } \\
\text { schläge } \\
\text { in } \\
\mathrm{mm}\end{array}$} & \multirow{3}{*}{$\begin{array}{c}\text { Regen } \\
\text { tage }\end{array}$} \\
\hline & \multicolumn{2}{|c|}{$\begin{array}{l}\text { Monats- } \\
\text { summe }\end{array}$} & \multicolumn{2}{|c|}{$\begin{array}{c}\text { Tages- } \\
\text { durchschnitt }\end{array}$} & \multirow{2}{*}{$\begin{array}{l}\text { Tage } \\
\text { ohne } \\
\text { Sonne }\end{array}$} & \multirow{2}{*}{$\begin{array}{c}\text { Monats- } \\
\text { summe der } \\
\text { Tagesmittel }\end{array}$} & \multirow{2}{*}{$\begin{array}{l}\text { Tagesmittel } \\
\text { des ganzen } \\
\text { Monats }\end{array}$} & & \\
\hline & Stdn. & Min. & Stdn. & Min. & & & & & \\
\hline $\begin{array}{l}\text { Mai : } \\
\text { Juni : } \\
\text { Juli : } \\
\text { August : } \\
\text { September } \\
\text { Oktober. }\end{array}$ & $\begin{array}{l}248 \\
236 \\
320 \\
234 \\
194 \\
162\end{array}$ & $\begin{array}{l}45 \\
50 \\
35 \\
40 \\
45 \\
40\end{array}$ & $\begin{array}{r}8 \\
7 \\
10 \\
7 \\
6 \\
5\end{array}$ & $\begin{array}{l}1 \\
54 \\
20 \\
34 \\
29 \\
15\end{array}$ & $\begin{array}{l}1 \\
3 \\
0 \\
0 \\
0 \\
0\end{array}$ & $\begin{array}{l}476.85 \\
526.85 \\
668.7 \\
589,3 \\
465,85 \\
418,9\end{array}$ & $\begin{array}{l}15.38 \\
17,56 \\
21,57 \\
19.07 \\
15,53 \\
13,51\end{array}$ & $\begin{array}{r}46.6 \\
6,9 \\
16.3 \\
86,5 \\
33,5 \\
4,9\end{array}$ & $\begin{array}{r}10 \\
7 \\
5 \\
12 \\
9 \\
4\end{array}$ \\
\hline
\end{tabular}

Bezüglich der Entwickelung der Reben im Berichtsjahre ist für die Pfalz nachstehendes zu bemerken:

Die Reben waren vom Vorjahre her gut durch den Winter gekommen und die Ausreife sowie die Menge des Tragholzes war überall eine befriedigende. Bei dem günstigen Frühjabrswetter konnten die notwendigen Rebarbeiten, wie namentlich das Düngen und der Rebschnitt so gefördert werden, daß sie trotz der infolge der Trockenheit erschwerten Bodenbearbeitung und trotz des Mangels an geschulten Arbeitskräften vor Eintritt der Vegetation noch rechtzeitig beendet werden konnten. Die jungen Knospen begannen sich bereits Mitte April zu entfalten, und der Austrieb erfolgte lückenlos. Am 16. April trat ein starker Kälterückschlag ein, und das Thermometer sank in den folgenden Nächten vielfach unter $0^{\circ}$. Diese Frostnächte wurden leider vielen bereits in der Entwickelung vorgeschrittenen Weinbergen wie namentlich den Portugieserreben verhängnisvoll und richteten durch Zerstörung der Angen sowohl in den Berglagen wie auch in den niederen Lagen recht beträchtlichen Schaden an, sodaß schon jetzt nicht mehr mit einem vollen Herbst gerechnet werden konnte. Das nach dem ersten Drittel des Monats Mai eintretende sehr günstige Wetter brachte die Entwickelung der Reben ungemein rasch voran und durch das Austreiben von Nebenaugen wurde der Frostschaden in manchen Lagen etwas herabgemildert. Der Samen* ansatz war in den nicht vom Frost beschädigten Lagen im allgemeinen ein befriedigender. Die Blüte begann in geschützten Lagen schon zu Anfang des Monats Juni und war in der Mitte dieses Monats fast allgemein. Gegen den 20. Juni trat wiederum eine empfindliche Abkühlung ein, die den Verlauf der Blüte, die in den kleineren Lagen noch im Rückstand war, beeinträchtigte und die Ausbreitung des Wurmes förderte. Auf den trockenen Juni folgte ein fast dürrer Juli. Obwohl die anhaltende Hitze die Verbreitung der Schädlinge hintanhielt, so wurde doch andererseits die Fortentwickelung der Trauben namentlich in den Berglagen und solchen mit sandigem Untergrund sehr gehemmt. Erst gegen Mitte August stellte sich der sehnsüchtig erwartete Regen ein, der dann im Verein mit der warmen Augustsonne die Entwickelung und das Wachstum der Trauben außerordentlich förderte und sie rasch der Vollreife entgegenführte. Auch der Monat September mit seinen sonnigen Tagen und seinen Niederschlägen brachte für die Weinberge den Abschlufo eines Witterungsverlaufs, wie er günstiger kaum bätte sein können.

Von den pflanzlichen und tierischen Schädlingen traten zwar Oidium und Peronospora schon früh auf, doch konnten beide Pilze infolge rechtzeitiger und energischer Bekämpfung durch Schwefeln und Spritzen, sowie dank der günstigen Witterungsverbältnisse nirgends einen nennenswerten Umfang annehmen. Dagegen verursachte der Heu- und Sauerwurm wiederum stellenweise einen nicht unbeträchtlichen Schaden. Trotz ergangener Warnungen vor einer Frühlese begann man schon am 10. September mit dem Portugieserherbst, der am 20. September bereits beendet war. Das Mengenergebnis war meist ein recht geringes, dagegen war die Qualität eine ganz ausge- 
zeichnete. Nach Beendigung der Rotweinlese begann man auch schon am 24. September mit der Lese der Weißrtrauben, die, wenn man von einigen größeren Gütern absieht, die zur Erzielung von Auslesen noch längere Zeit zuwarteten, bis Mitte Oktober dauerte und bei herrlichstem Sommerwetter sich vollzog. Wie zu erwarten stand, war die Güte der Trauben eine ganz hervorragende und zeigten die Moste selbst aus kleinen Lagen eine große liebliche Süße und ein hervorragendes Bukett. Der Ertrag war infolge der Frostschäden ein ganz unterschiedlicher, und man kann das Gesamtmengenergebnis wohl durchschnittlich noch als eine schwache halbe Ernte bezeichnen.

Was die Preisbeurteilung anbetrifft, so kostete zu Beginn der Lese Portugiesermost pro Logel (40 l) 150-300 M., Weißmost 200-1500 M. Unter dem Einflusse der schlechten Valuta und ausländischer preistreibender Käufer (Schweiz, Holland, Belgien) steigerten sich aber die Preise bis zu nie dagewesenen Sätzen. Je nach Qualität kostet jetzt das Liter Portugieser 8-14 M. und das Liter Weißmost 12-50 M.

Die Zahl der untersuchten Moste betrug 354. Von diesen entfallen auf Weißmost 302 und auf Rotmost 52 Proben, die sich auf die einzelnen Weinbaugebiete der Pfala, wie folgt, verteilen:

Tabelle I. Art und Zahl der untersuchten Moste.

\begin{tabular}{|c|c|c|c|c|c|c|c|c|c|c|c|c|}
\hline \multirow{2}{*}{$\begin{array}{c}\text { Art } \\
\text { des } \\
\text { Mostes }\end{array}$} & \multicolumn{2}{|c|}{$\begin{array}{l}\text { Ober- } \\
\text { Haardt }\end{array}$} & \multicolumn{2}{|c|}{$\begin{array}{l}\text { Mittel- } \\
\text { Haardt }\end{array}$} & \multicolumn{2}{|c|}{$\begin{array}{l}\text { Unter- } \\
\text { Haardt }\end{array}$} & \multicolumn{2}{|c|}{$\begin{array}{c}\text { Alsenz-, Eis. } \\
\text { und Gluntal } \\
\text { (Nordpfalz) }\end{array}$} & \multicolumn{2}{|c|}{$\begin{array}{l}\text { Übrige Pfalz } \\
\text { (Rheinebtne) }\end{array}$} & \multicolumn{2}{|c|}{ Im ganzen } \\
\hline & \begin{tabular}{|c|} 
Zahl der \\
Proben
\end{tabular} & $\%$ & $\begin{array}{c}\text { Zahl der } \\
\text { Proben }\end{array}$ & $\%$ & $\begin{array}{c}\text { Zahl der } \\
\text { Proben }\end{array}$ & $\%$ & $\begin{array}{c}\text { Zahl der } \\
\text { Proben }\end{array}$ & $\%$ & $\begin{array}{c}\text { Zahl der } \\
\text { Proben }\end{array}$ & $\%$ & $\begin{array}{c}\text { Zahl der } \\
\text { Proben }\end{array}$ & $\%$ \\
\hline $\begin{array}{l}\text { Rotmost. } \\
\text { Weikmost }\end{array}$ & $\begin{array}{r}20 \\
147\end{array}$ & $\begin{array}{r}5,7 \\
41,5\end{array}$ & $\begin{array}{l}25 \\
58\end{array}$ & $\begin{array}{r}7,1 \\
16,4\end{array}$ & $\begin{array}{r}3 \\
24\end{array}$ & $\begin{array}{l}0,8 \\
6,9\end{array}$ & $\begin{array}{r}3 \\
58\end{array}$ & $\begin{array}{r}0,8 \\
16.4\end{array}$ & $\begin{array}{r}1 \\
15\end{array}$ & $\begin{array}{l}0,3 \\
4.2\end{array}$ & $\begin{array}{r}52 \\
302\end{array}$ & $\begin{array}{l}14,7 \\
85,3\end{array}$ \\
\hline Zusammen: & 167 & 47,2 & 83 & 23,5 & 27 & 7,6 & 61 & 17,2 & 16 & 4,5 & 354 & 100,0 \\
\hline
\end{tabular}

Tabelle II. Mostgewioht e.

a) Rotmoste.

\begin{tabular}{|c|c|c|c|c|c|c|c|c|c|c|c|c|}
\hline \multirow{2}{*}{$\begin{array}{c}\text { Most- } \\
\text { gewichte } \\
\text { in } \\
\text { Oechsle- } \\
\text { Graden }\end{array}$} & \multicolumn{2}{|c|}{$\begin{array}{l}\text { Ober- } \\
\text { Haardt }\end{array}$} & \multicolumn{2}{|c|}{$\begin{array}{l}\text { Mittel- } \\
\text { Haardt }\end{array}$} & \multicolumn{2}{|c|}{$\begin{array}{l}\text { Unter- } \\
\text { Haardt }\end{array}$} & \multicolumn{2}{|c|}{$\begin{array}{l}\text { Alsenz-, Eis- } \\
\text { und Glantal } \\
\text { (Nordpfal } \mathrm{C} \text { ) }\end{array}$} & \multicolumn{2}{|c|}{$\begin{array}{l}\text { Ubrige Pfalz } \\
\text { (Rheinebene) }\end{array}$} & \multicolumn{2}{|c|}{ Im ganzen } \\
\hline & $\begin{array}{l}\text { Zahl der } \\
\text { Yroben }\end{array}$ & $\%$ & $\begin{array}{l}\text { Zahl der } \\
\text { Proben }\end{array}$ & $\%$ & $\begin{array}{c}\text { Zabl der } \\
\text { Proben }\end{array}$ & $\%$ & $\begin{array}{c}\text { Zahl der } \\
\text { Proben }\end{array}$ & $\%$ & $\mid \begin{array}{c}\mathrm{Zahl} \text { der } \\
\text { Proben }\end{array}$ & $\%$ & $\begin{array}{l}\text { Zahl der } \\
\text { Proben }\end{array}$ & $\%$ \\
\hline $60-69,9$ & 1 & 1,9 & - & - & - & - & - & - & - & - & 1 & 1,9 \\
\hline $70-79,9$ & 5 & 9,6 & 4 & 7,7 & 1 & 1,9 & - & - & 1 & 1,9 & 11 & 21,2 \\
\hline $80-89,9$ & 13 & 25,0 & 18 & 34,6 & 2 & 3,9 & 3 & 5,8 & - & - & 36 & 69.2 \\
\hline $90-99.9$ & 1 & 1,9 & 3 & 5.8 & - & - & - & - & - & - & 4 & 7,7 \\
\hline Zusammen: & 20 & 38,4 & 25 & 48,1 & 3 & 5,8 & 3 & 5,8 & 1 & 1,9 & 52 & 100,0 \\
\hline
\end{tabular}

b) Weifmoste.

\begin{tabular}{r|r|r|r|r|r|r|r|r|r|r|r|r|}
$60-69,9$ & 8 & 2,6 & - & - & - & - & - & - & 1 & 0,35 & 9 & 3,0 \\
$70-79,9$ & 37 & 12,2 & - & - & 1 & 0,3 & 2 & 0,7 & 3 & 1,0 & 43 & 14,2 \\
$80-89,9$ & 79 & 26,2 & 2 & 0,7 & 6 & 2,0 & 13 & 4,3 & 8 & 2,6 & 108 & 35,8 \\
$90-99,9$ & 18 & 6,0 & 15 & 4,9 & 7 & 2,4 & 20 & 6,6 & 2 & 0,7 & 62 & 20,5 \\
$100-109,9$ & 5 & 1,6 & 14 & 4,6 & 9 & 3,0 & 23 & 7,6 & 1 & 0,35 & 52 & 17,2 \\
$110-119,9$ & - & - & 21 & 7,0 & 1 & 0,3 & - & - & - & - & 22 & 7,3 \\
$120-129,9$ & - & - & 6 & 2,0 & - & - & - & - & - & - & 6 & 2,0 \\
\hline Zusammen: & 147 & 48,6 & 58 & 19,2 & 24 & 8,0 & 58 & 19,2 & 15 & 5,0 & 302 & 100,0
\end{tabular}


Tabelle III. Freie Säuren.

a) Rotmoste.

\begin{tabular}{|c|c|c|c|c|c|c|c|c|c|c|c|c|}
\hline \multirow{2}{*}{$\begin{array}{c}\text { Freie } \\
\text { Säuren } \\
\text { g in } \\
100 \text { ccm }\end{array}$} & \multicolumn{2}{|c|}{$\begin{array}{l}\text { Ober- } \\
\text { Haardt }\end{array}$} & \multicolumn{2}{|c|}{$\begin{array}{l}\text { Mittel- } \\
\text { Haardt }\end{array}$} & \multicolumn{2}{|c|}{$\begin{array}{l}\text { Unter: } \\
\text { Haardt }\end{array}$} & \multicolumn{2}{|c|}{$\begin{array}{c}\text { Alsenz*, Eis- } \\
\text { und Glantal } \\
\text { (Nordpfalz) }\end{array}$} & \multicolumn{2}{|c|}{$\begin{array}{l}\text { Ubrige Pfalz } \\
\text { (Kheinebene) }\end{array}$} & \multicolumn{2}{|c|}{ Im ganzen } \\
\hline & $\begin{array}{c}\text { Zahl der } \\
\text { Proben }\end{array}$ & $\%$ & $\left|\begin{array}{c}\text { Zahl der } \\
\text { Proben }\end{array}\right|$ & $\%$ & $\begin{array}{c}\text { Zabl der } \\
\text { Prohen }\end{array}$ & $\%$ & $\begin{array}{c}\text { Zabl der } \\
\text { Proben }\end{array}$ & $\%$ & $\begin{array}{c}\text { Zahl der } \\
\text { Proben }\end{array}$ & $\%$ & $\mid \begin{array}{c}\text { Zahl der } \\
\text { Proben }\end{array}$ & $\%$ \\
\hline $0,50-0,59$ & 1 & 1,9 & 8 & 15,4 & - & - & 2 & 8,9 & - & - & 11 & 21,2 \\
\hline $0,60-0,69$ & 2 & 3,9 & 10 & 19,3 & - & - & 1 & 1,9 & - & - & 13 & 25,0 \\
\hline $0,70-0,79$ & 7 & 13,3 & 5 & 9,6 & 2 & 3,9 & - & - & - & - & 14 & 26,9 \\
\hline $0,80-0,89$ & 3 & 5,8 & 1 & 1,9 & - & - & - & - & - & - & 4 & 7,7 \\
\hline $0,90-0,99$ & 4 & 7,7 & 1 & 1,9 & - & - & - & - & - & - & 5 & 9,6 \\
\hline $1,00-1,19$ & 3 & 5,8 & - & - & 1 & 1,9 & - & - & - & - & 4 & 7,7 \\
\hline $1,70-1,79$ & - & - & - & - & - & - & - & - & 1 & 1,9 & 1 & 1,9 \\
\hline Zusammen: & 20 & 38,4 & 25 & 48,1 & 3 & 5,8 & 3 & 5,8 & 1 & 1,9 & 52 & 100,0 \\
\hline \multicolumn{13}{|c|}{ b) Weifmoste. } \\
\hline $0,50-0,59$ & - & - & 20 & 6,6 & 3 & 1,0 & 12 & 4,0 & - & - & 35 & 11,7 \\
\hline $0,60-0,69$ & 10 & 3,3 & 25 & 8,3 & 1 & 0,3 & 25 & 8,3 & - & - & 61 & 20,2 \\
\hline $0,70-0,79$ & 27 & 8,9 & 8 & 26 & 8 & 2,7 & 15 & 4,9 & 1 & 0,3 & 59 & 19,5 \\
\hline $0,80-0,89$ & 30 & 9,9 & 2 & 0,7 & 5 & 1,6 & 3 & 1,0 & 2 & 0,7 & 42 & 13,9 \\
\hline $0,90-0,99$ & 37 & 12,3 & 1 & 0,3 & 3 & 1,0 & 1 & 0,3 & $\mathbf{3}$ & 1,0 & 45 & 14,9 \\
\hline $1,00-1,19$ & 36 & 11,9 & 2 & 0,7 & 2 & 0,7 & 2 & 0,7 & 4 & $1, \mathbf{3}$ & 46 & 15,2 \\
\hline $1,20-1,39$ & 5 & 1,6 & - & - & 2 & 0,7 & - & - & 3 & 1.0 & 10 & 3,3 \\
\hline $1,40-1,59$ & 5 & 1.6 & - & - & 2 & 0.7 & - & - & 2 & 0.7 & 4 & 1.3 \\
\hline Zusammen: & 147 & 48,6 & 58 & 19,2 & 24 & 8,0 & 58 & 19,2 & 15 & 5,0 & 302 & 100,0 \\
\hline
\end{tabular}

Tabelle IV. Schwankungen und Mittel der Mostgewichte.

\begin{tabular}{|c|c|c|c|c|c|}
\hline $\begin{array}{l}\text { Oechsle } \\
\text { Grade }\end{array}$ & $\begin{array}{l}\text { Ober- } \\
\text { Haardt }\end{array}$ & $\begin{array}{l}\text { Mittel- } \\
\text { Haardt }\end{array}$ & $\begin{array}{l}\text { Notmos } \\
\text { Unter-" } \\
\text { Haardt }\end{array}$ & $\begin{array}{l}\text { Alsenz-, Eis- und } \\
\text { Glantal (Nordpfalz) }\end{array}$ & $\begin{array}{l}\text { Übrige Pfalz } \\
\text { (Rheinebene) }\end{array}$ \\
\hline Höchst & $97^{\circ}$ & $93^{\circ}$ & $88^{\circ}$ & $88^{\circ}$ & $\left.77^{01}\right)$ \\
\hline Mindest & $60^{\circ}$ & $74^{\circ}$ & $74^{0}$ & $80,5^{\circ}$ & - \\
\hline Mittel & $80,9^{\circ}$ & $84,5^{0}$ & $80,5^{\circ}$ & $85,1^{0}$ & - \\
\hline \multicolumn{6}{|c|}{ b) Weißßmoste. } \\
\hline Mindest & $60^{\circ}$ & $85^{\circ}$ & $77^{\circ}$ & $75^{\circ}$ & $66^{\circ}$ \\
\hline Mittel & $82,2^{\circ}$ & $105,2^{\circ}$ & $95,5^{\circ}$ & $95,2^{\circ}$ & $75,9^{\circ}$ \\
\hline
\end{tabular}

Tabelle V. Schwankungen und Mittel des Gehaltes an freier Säure (g im Liter).

$\begin{array}{lccccc}\text { Höchst } & 11,25 & 9,00 & \text { a) Rotmoste. } & 10,5 \\ \text { Mindest } & 5,85 & 5,40 & 7,80 & 6,6 & \left.17,1^{1}\right) \\ \text { Mittel } & 8,22 & 6,52 & 8,70 & 5,40 & - \\ & & & \text { b) Weifmoste. } & \\ \text { Höchst } & 14,55 & 10,7 & 12,9 & 11,4 & 14,25 \\ \text { Mindest } & 6,0 & 5,25 & 5,55 & 4,95 & 7,35 \\ \text { Mittel } & 9,11 & 6,50 & 8,32 & 6,75 & 11,0\end{array}$

1) Amerikanerrebe, seg. Killiansrebe in Dadenhofen. 
Die ermittelten Mostgewichte und Säuregrade sind aus den Tabellen II und III zu erseben, während die Tabellen IV und V über die beobachteten Höchst- und Mindestgewichte bezw. die Höchst und Mindestgebalte an Säure Aufschluß geben.

Hiernach ist bei den Rotmosten das durchschnittliche Mostgewicht für die Oberhaardt $80,9^{\circ}$, Mittelhaardt $84,5^{\circ}$, Unterhaardt $80,5^{\circ}$, Alsenz , Eis- und Glantal $85,1^{\circ}$ und für die übrige Pfalz (Rheinebene) $77^{\circ}$. Der durchschnittliche Säuregehalt beträgt 8,22 bezw. $6,52,8,70$ und $5,90 \%$.

Der in der Tabelle $V$ festgestellte abnorm hohe Säuregehalt einer Probe mit $17,7 \%$ bezieht sich nicht auf eine einheimische Rebe, sondern auf eine Amerikanerrebe (sog. Kiliansrebe) der Gemarkung Dudenbofen. Auch dieser Befund spricht wieder zur Genüge dafür, daßo die Kiliansrebe auch in Jahren mit großer Reife nicht befähigt ist, ein konsumfäbiges Getränk zu liefern und daher vor einem weiteren Anbau dieser Rebe nur dringend gewarnt werden muB.

Bei den Weifmosten beträgt das durchschnittliche Mostgewicht für die Oberhaardt $83,2^{\circ}$, Mittelhaardt $105,2^{\circ}$, Unterhaardt $95,5^{\circ}$, Alsenz-, Eis- und Glantal $95,2^{0}$ und für die übrige Pfalz (Rheinebene) $75,9^{\circ}$. Der durchschnittliche Sâuregehalt beträgt 9,11 bezw. $6,50,8,32,6,75$ und $11,0 \%$.

Auch in diesen Zahlen kommt das Kriterium eines reifen Jahrgangs, nämlich sehr bohes Mostgewicht und niedrige freie Säure treffend zum Ausdruck und es ist insbesondere bemerkenswert, $\mathrm{daB}$ von den untersucbten Proben kein einziger Most ein Mostgewicht unter $60^{\circ}$ Oechsle zeigt und nur 10 Proben $(2,8 \%)$ ein Mostgewicht von weniger als $70^{\circ}$ hatten. Eine Zuckerung kann sonach im Hinblick auf die Bestimmungen des Weingesetzes weder für die Weißmoste noch für die Rotmoste in irgend einer Weise für die Pfalz zugestanden werden, sie wäre auch unsinnig, da sie die Weine brandig und unbarmonisch machen würde. Die 1921-er können somit nur als Naturweine in den Handel kommen.

Groß, teilweise sogar sehr groß sind die Spätlesen und Auslesen der besten Lagen unserer großen Weingüter, die in der vorstebenden Statistik keine Aufnahme gefunden haben. Es wurden hier vielfach Oechslegewichte von $150-251^{\circ}$ erzielt. Wegen ihrer hervorragenden Güte können sonach die 1:21-er Moste den hervorragenden Jahren 1904 und 1911 als völlig ebenbürtig zur Seite gestellt werden und dürfte sogar der beurige Jahrgang in bezug auf Art und Fülle jene noch übertreffen.

Die Gärung setzte bei der hohen Lesetemperatur sehr rasch ein und verlief meist auferordentlich schnell und stümmisch. Viele 1921.er sind bereits so hell, dab sie schon als fast völlig klare Weine verzapft werden können. Selbst Moste mit $100^{\circ}$ Oechsle und mehr sind schon ganz bis auf kleine Zuckerreste vergoren. Nach. den bis jetzt von uns angestellten Untersuchungen sind die 1921-er Weine im Gegensatz zu den 1911-er Gewächsen keineswegs arm an Extrakt, und es ist ganz besonders die Tatsache bemerkenswert, daß die Hefe in diesem Jahre befähigt war, in manchen sehr zuckerreichen Mosten von 130-140 Oechsle den Zucker glatt zu vergären und so große Mengen an Alkohol zu erzeugen, wie wir sie bis jetzt noch in keinem Jahrgang zu beobachten Gelegenheit hatten. So zeigten beispielsweise zwei Weine aus Deidesheim (Forster Langenacker und Forster Straße) kurz nach Verlauf der stürmischen Gärung 12,11 Gew. $\% \%=15,26$ Vol. $\%$ bezw. 12,65 Gew.. $\%=15,95$ Vol. $\%$ \% Alkohol bei noch 0,23 bezw. $0,76 \mathrm{~g}$ vorhandenem Zucker. Diese Mehrleistung der Hefe im Vergleich zu anderen Jahren läßt sich wohl nur dadurch erklären, daß die Lebenstätigkeit der betreffenden Hefen nicht dureh die Giftwirkungen der Edelfäule (Botrytis) oder anderer Organismen sehädlich beeinflußt worden ist, und auch hierin kommt somit wieder die gesunde und reife Art des Jahrgangs 1921 deutlich zum Ausdruck.

Was nun die Behandlung des Neuen betrifft, so sei für die Praxis nur kurz auf folgendes hingewiesen: Die Moste sind fast ausnahmsweise arm an Säure, und es 
muB daher das Bestreben der Kellerbehandlung darauf gerichtet sein, den Weinen die noch vorhandene geringe Menge an Äpfelsäure nach Möglichkeit zu erhalten, denn eine gewisse Säure ist für den harmonischen Geschmack notwendig und ausschlaggebend. Es ist schon oft hervorgehoben worden, daß auf den Säureabbau fördernd wirke vor allem das Lagern bei einer hohen Kellertemperatur, das Aufrühren der Hefe, sowie ein längeres Liegenlassen des Jungweins auf der Hefe. Wir müssen daher in diesem Jahre gerade die entgegengesetzten Maßnahmen zur Erhaltung der Säure treffen. Der Keller, in dem die Weine völlig vergoren sind, darf sonach nicht mehr geheizt werden, und eine Temperatur von $12^{\circ} \mathrm{C}$ ist als genügend zu betrachten. Das Aufrühren der Hefe ist zu unterlassen, und der Abstich der Jungweine, sofern es sich nicht um schwere hochwertige Moste handelt, ist so frühzeitig als möglich vorzunehmen. Vielfach wurden uns Proben von Jungweinen überbracht, die wegen ihres mehr oder weniger süßen Geschmacks den Verdacht nahelegten, daß sie in der Gärung stecken geblieben seien. Wir konnten aber in allen Fällen einen Gehalt von etwa 9;5 g und mehr Alkohol in $100 \mathrm{cem}$ feststellen bei meist noch $0,5-2,0 \%$ Zucker. Von einem Steckenbleiben in der Gärung kann sonach nicht gesprochen werden, gleichwohl haben wir aber in allen diesen Fällen ein nochmaliges Aufrähren der Hefe empfohlen, um den noch vorhandenen Zucker soweit als möglich zur Vergärung zu bringen und ein späteres Umschlagen der Weine beim weiteren Ausbau nach Möglichkeit hintanzubalten.

Gerade wegen der bei einem großen Teile der pfälzischen 1921 er Weine noch vorhandenen Süße bedarf aber der Neue bei der Erziehung zur Flaschenreife einer besonders sorgtältigen Kellerbehandlung, wenn er nicht zu einem Sorgenkind für den Besitzer werden soll. In zweifelhaften Fällen haben wir daher um Einsendung einer Probe an die Anstalt gebeten.

Eine weitere Eigentümlichkeit der 1921-er Jungweine ist ihre starke Neigung zum Rahn- oder Fuchsigwerden. Schon nach kurzem Stehen an der Luft werden sie vielfach tiefbraun. Es ist diese Erscheinung auf die Wirkung eines namentlich in überreifen und edelfaulen Trauben vorhandenen Enzyms, der sog. Oxydase zurückzuführen, de befähigt ist, den Sauerstoff der Luft auf gewisse Bestandteile des Weines zu übertragen, der hierbei eine starke braune Färbung annimmt. Ein geeignetes Mittel zur Verhütung des Rahnwerdens bietet die schweflige Säure. Solchen zum Braunwerden neigenden Jungweinen gibt man nach dem ersten Abstich pro Fuder etwa 10 Stück der dünnen nicht abtropfenden Schwefelschnitte. Eine starke Schwefelgabe ist in diesem Jahre ohnedies schon mit Rücksicht auf die Erhaltung der Säure geboten.

Bei einem Rückblick auf das Weinjahr 1921 hat zwar infolge der Frostschäden die Menge des Ertrages wenig befriedigt, um so mehr dagegen die Güte desselben, und in der Geschichte des Weinbaues wird der 1921-er Jahrgang stets einen Ehrenplatz einnehmen und als ein großer genannt werden. Wie fast in jedem Jahr, so mußte auch hener wieder wahrgenommen werden, daf in manchen Orten des oberen Gebirgeș der Beginn der Lese wieder ein viel zu früher war. Ganz abgesehen davon, daß sich beuer die Winzer bei den anfänglich niederen Preisen dadurch selbst geschädigt haben, war hierzu auch um so weniger Anlaß gegeben, als das Wetter so günstig war, daß uns täglich höhere Mostgewichte von der Natur von selbst aufgedrängt wurden. Möge daher doch künftig beachtet werden, daß der deutsche Weinbau in mancher Hinsicht großen Gefahren und Schädigungen entgegengeht und nur bestehen kann, wenn jedes Weinbaugebiet bemüht ist, das beste an Qualität zu erreichen, was unter den gegebenen Verhältnissen möglich ist. 